\title{
Barrier Options under Lévy Processes: a Short-Cut
}

\author{
José Fajardo* \\ November 7, 2018
}

\begin{abstract}
In this paper we present a simple way to price a class of barrier options when the underlying process is driven by a huge class of Lévy processes. To achieve our goal we assume that our market satisfies a symmetry property. In case of not satisfying that property some approximations and relationships can be obtained.
\end{abstract}

Keywords: Barrier Options; Lévy Processes; Implied volatility; Market Symmetry.

JEL Classification: C52; G10

\section{Introduction}

Recently, the relationship between the implied volatility symmetry and other symmetry concepts has been established, as Fajardo and Mordecki (2006) have shown for Lévy process and Carr and Lee (2009) have shown for lo$\mathrm{cal} /$ stochastic volatility models and time-changed Lévy processes. Also, Fajardo and Mordecki (2012) have studied the relationship of symmetry properties with the skewness premium. Moreover, it is known that important applications, such as the construction of semi-static hedges for exotic options can be obtained, as Carr, Ellis, and Gupta (1998) and Carr and Lee (2009)

*Brazilian School of Public and Business Administration, Getulio Vargas Foundation, Praia de Botafogo 190, 22253900 - Rio de Janeiro, RJ, Brazil. E-mail address: jose.fajardo@fgv.br 
have shown, and its extension to multivariate derivatives due to Molchanov and Schmutz (2010).

The importance of such symmetry properties have demanded the analysis of conditions to verify what kind of underlying processes satisfy such properties, as Carr and Lee (2009), Fajardo and Mordecki (2008), Fajardo and Mordecki (2010) and Tehranchi (2009) results have shown.

On the other hand, Bates (1997) verified that put-call symmetry does not hold in practice, by constructing a hypothesis test that compares the observed relative call-put prices and the ratio given by Bates' rule. The absence of symmetry has also been reported by Carr and $\mathrm{Wu}(2007)$. They found asymmetric implied volatility smiles in currency options. But, besides these empirical findings other applications of symmetry properties can justify their use, as for example the pricing of credit instruments, such as barrier contracts, as Carr and Lee (2009) have shown by constructing semi-static hedging for a class of barrier options.

In the context of Lévy processes the pricing of barrier contracts is a delicated issue. We find some contributions, as Lipton (2002) who considers jump-diffusion process with exponentially distributed jumps. Also, Kou and Wang (2004) price barrier option with the double exponential jump-diffussion model. Asmussen, Madan, and Pistorius (2007) consider the CGMY model to price some kind of barrier options. For more general Lévy processes we have the contributions of Rogers (2000), for spectrally one-sided Lévy processes, and Asmussen, Avram, and Pistorius (2004), for phase-type jumps. More recently, Fourier transform methods have been used to price barrier options under more general Lévy processes see Eberlein, Glau, and Papapantoleon (2010), Eberlein, Glau, and Papapantoleon (2011) and Carr and Crosby (2010). For more references see Schoutens and Cariboni (2009).

In this paper we present a very simple way to price special kinds of exotic options, such as digital call and put options, asset-or-nothing options, under a huge family of Lévy processes assuming that a symmetry property holds. When symmetry property does not hold we obtain some approximations and we price the down-and-in power option, by extending Th. 7.6 in Carr and Lee (2009) for our class of Lévy processes. 
The paper is organized as follows, in Section 2 we introduce our model. In Section 3 we present our main results for symmetric markets. In Section 4 we present the results under absence of symmetry. In Section 5 we present some numerical examples and the last section concludes.

\section{Market Model}

Consider a real valued stochastic process $X=\left\{X_{t}\right\}_{t \geq 0}$, defined on a stochastic basis $\mathcal{B}=\left(\Omega, \mathcal{F}, \mathbf{F}=\left(\mathcal{F}_{t}\right)_{t \geq 0}, \mathbb{Q}\right)$, being càdlàg, adapted, satisfying $X_{0}=0$, and such that for $0 \leq s<t$ the random variable $X_{t}-X_{s}$ is independent of the $\sigma$-field $\mathcal{F}_{s}$, with a distribution that only depends on the difference $t-s$. Assume also that the stochastic basis $\mathcal{B}$ satisfies the usual conditions (see Jacod and Shiryaev (1987)). The process $X$ is a Lévy process, and is also called a process with stationary independent increments. For Lévy process in Finance see Schoutens (2003) and Cont and Tankov (2004).

In order to characterize the law of $X$ under $\mathbb{Q}$, consider, for $q \in \mathbb{R}$ the Lévy-Khinchine formula, that states

$$
\mathbf{E} e^{i q X_{t}}=\exp \left\{t\left[i a q-\frac{1}{2} \sigma^{2} q^{2}+\int_{\mathbb{R}}\left(e^{i q y}-1-i q h(y)\right) \Pi(d y)\right]\right\},
$$

with

$$
h(y)=y \mathbf{1}_{\{|y|<1\}}
$$

a fixed truncation function, $a$ and $\sigma \geq 0$ real constants, and $\Pi$ a positive measure on $\mathbb{R} \backslash\{0\}\}^{1}$ such that $\int\left(1 \wedge y^{2}\right) \Pi(d y)<+\infty$, called the Lévy measure. The triplet $\left(a, \sigma^{2}, \Pi\right)$ is the characteristic triplet of the process, and completely determines its law.

Now we use the extension to the complex plane used by Lewis $(2001)$, that is we define the Lévy-Khinchine formula in the $\operatorname{strip}\{z: a<\operatorname{Im}(z)<b\}$ where $a \leq-1$ and $b \geq 0$. Then we can define the characteristic exponent of the process $X$, in this strip, by:

$$
\psi(z)=a z i-\frac{1}{2} \sigma^{2} z^{2}+\int_{\mathbb{R}}\left(e^{i z y}-1-i z h(y)\right) \Pi(d y)
$$

\footnotetext{
${ }^{1} \Pi(\{0\})$ could be defined as 0 . Here we follows Cont and Tankov $(2004)$.
} 
this function $\psi$ is also called the cumulant of $X$, having $\mathbf{E}\left|e^{z X_{t}}\right|<\infty$ for all $t \geq 0$, and $\mathbf{E} e^{z X_{t}}=e^{t \psi(z)}$. For $t=1$, Formula (2) reduces to exponent of eq. (1) with $\operatorname{Im}(z)=0$.

\subsection{Lévy market}

By a Lévy market we mean a model of a financial market with two assets: a deterministic savings account $B=\left\{B_{t}\right\}_{t \geq 0}$, with

$$
B_{t}=e^{r t}, \quad r \geq 0,
$$

where we take $B_{0}=1$ for simplicity, and a stock $S=\left\{S_{t}\right\}_{t \geq 0}$, with random evolution modeled by

$$
S_{t}=S_{0} e^{X_{t}}, \quad S_{0}=e^{x}>0,
$$

where $X=\left\{X_{t}\right\}_{t \geq 0}$ is a Lévy process.

In this model we assume that the stock pays dividends, with constant rate $\delta \geq 0$, and that the given probability measure $\mathbb{Q}$ is the chosen equivalent martingale measure. In other words, prices are computed as expectations with respect to $\mathbb{Q}$, and the discounted and reinvested process $\left\{e^{-(r-\delta) t} S_{t}\right\}$ is a $\mathbb{Q}$-martingale.

In terms of the characteristic exponent of the process this means that

$$
\psi(1)=r-\delta,
$$

based on the fact, that $\mathbf{E} e^{-(r-\delta) t+X_{t}}=e^{-t(r-\delta-\psi(1))}=1$, and condition (4) can also be formulated in terms of the characteristic triplet of the process $X$ as

$$
a=r-\delta-\sigma^{2} / 2-\int_{\mathbb{R}}\left(e^{y}-1-\mathbf{1}_{\{|y|<1\}}\right) \Pi(d y) .
$$

Then,

$$
\psi(z)=i z\left(r-\delta-\frac{\sigma^{2}}{2}\right)-z^{2} \frac{\sigma^{2}}{2}+\int_{-\infty}^{+\infty}\left[i z\left(1-e^{y}\right)+\left(e^{i z y}-1\right)\right] \Pi(d y)
$$

Henceforth we will denote this exponent by $\psi_{\beta}$ due to its future dependence on parameter $\beta$ of our jump structure. 


\subsection{Market Symmetry}

Here we use the symmetry concept introduced in Fajardo and Mordecki (2006). We define a Lévy market to be symmetric when the following relation holds:

$$
\mathcal{L}\left(e^{-(r-\delta) t+X_{t}} \mid \mathbb{Q}\right)=\mathcal{L}\left(e^{-(\delta-r) t-X_{t}} \mid \tilde{\mathbb{Q}}\right),
$$

meaning equality in law. Otherwise we call the Lévy market asymmetric. As Fajardo and Mordecki (2006) pointed out, a necessary and sufficient condition for (7) to hold is

$$
\Pi(d y)=e^{-y} \Pi(-d y) .
$$

This ensures $\tilde{\Pi}=\Pi$, and from this follows $a-(r-\delta)=\tilde{a}-(\delta-r)$, giving (7), as we always have $\tilde{\sigma}=\sigma$.

Moreover, in Lévy markets with jump measure of the form

$$
\Pi(d y)=e^{\beta y} \Pi_{0}(d y),
$$

where $\Pi_{0}(d y)$ is a symmetric measure, i.e. $\Pi_{0}(d y)=\Pi_{0}(-d y)$ and $\beta$ is a parameter that describe the asymmetry of the jumps, everything with respect to the risk neutral measure $\mathbb{Q}$.

As a consequence of (8), Fajardo and Mordecki (2006) found that the market is symmetric if and only if $\beta=-1 / 2$.

Now substituting in eq. (6), we observe the dependence of the characteristic exponent on the parameter $\beta$ :

$$
\psi_{\beta}(z)=i z\left(r-\delta-\frac{\sigma^{2}}{2}\right)-z^{2} \frac{\sigma^{2}}{2}+\int_{-\infty}^{+\infty}\left[i z\left(1-e^{y}\right)+\left(e^{i z y}-1\right)\right] e^{\beta y} \Pi_{0}(d y),
$$

from her we obtain the partial derivative with respect to $\beta$ :

$$
\frac{\partial \psi_{\beta}}{\partial \beta}(-z)=\int_{-\infty}^{+\infty}\left[\left(e^{-i z y}-1\right)-i z\left(1-e^{y}\right)\right] y e^{\beta y} \Pi_{0}(d y) .
$$

It will be needed in the approximations to be presented later. 


\section{Results}

Let $\sigma_{i m p}(x)(x, \beta)$ denote the Black-Scholes implied volatility, that depends on the $\log$-moneyness $x=\log K / F$, where $K$ is the strike price and $F$ the

forward price, the symmetry parameter $\beta$ and denote by $f_{x}$ the price of a European style digital call option with maturity $T$ and barrier $K_{x}$, i.e. at Maturity derivative pays off $f_{x}(y)=1_{\left\{e^{y}>K_{x}\right\}}$. The put version is denoted by $g_{x}$ and pay off given by $g_{x}(y)=1_{\left\{e^{y} \leq K_{x}\right\}}$. As in Eberlein, Glau, and Papapantoleon (2010), we can take $y=X_{T}+\ln \left(S_{0}\right)+(r-\delta) T$, then $f_{0}\left(X_{T}\right)=$ $1_{\left\{e^{\left.X_{T}>1\right\}}\right.}$. Now our main result.

Theorem 3.1. When symmetry holds $(\beta=-1 / 2)$ the price of the digital call option with barrier $K_{0}$ is given by:

$$
f_{0}=e^{-r T} N\left(-\frac{\sigma \sqrt{T}}{2}\right)
$$

In the case of an European digital put option, the price is given by:

$$
g_{0}=e^{-r T}\left[1-N\left(-\frac{\sigma \sqrt{T}}{2}\right)\right]
$$

Proof. Lets denote $B S_{c}\left(\sigma_{i m p}(x, \beta)\right)$ the Black and Scholes price of and European call with maturity $T$, strike $K$ and spot price $S_{0}$. When the risk neutral interest rate is denoted by $r$ and the price is driven by a Lévy process with characteristic triplet $(a, \sigma, \Pi)$, that $\mathrm{BS}$-price is given by:

$$
B S_{c}\left(\sigma_{i m p}(x, \beta)\right)=\mathrm{Ee}^{-\mathrm{rT}}\left(\mathrm{S}_{0} \mathrm{e}^{\mathrm{X}_{\mathrm{T}}}-\mathrm{K}\right)^{+} .
$$

Now, we know by Lévy-Khintchine formula

$$
\psi_{\beta}(z)=i z a-z^{2} \frac{\sigma^{2}}{2}+\int_{-\infty}^{+\infty}\left(e^{i z y}-1\right) e^{\beta y} \Pi_{0}(d y),
$$

when $\Pi(d y)=e^{\beta y} \Pi_{0}(d y)$. Also, as we are interested in properties of option prices we need that discounted prices process be martingales, then as showed in (6), characteristic exponent must be given by:

$$
\psi_{\beta}(z)=i z\left(r-\delta-\frac{\sigma^{2}}{2}\right)-z^{2} \frac{\sigma^{2}}{2}+\int_{-\infty}^{+\infty}\left[i z\left(1-e^{y}\right)+\left(e^{i z y}-1\right)\right] e^{\beta y} \Pi_{0}(d y) .
$$


Then, we can compute the BS-price as:

$$
\begin{aligned}
B S_{c}\left(\sigma_{i m p}(x, \beta)\right) & =\int_{-\infty}^{+\infty} e^{-r T}\left(S_{0} e^{x}-K\right)^{+} f_{X_{T}}(x) d x \\
& =\frac{e^{-r T}}{2 \pi} \int \frac{-K^{i z+1} e^{-i z\left(\ln \left(S_{0}\right)+(r-q) T\right)}}{z(z-i)} e^{T \psi_{\beta}(-z)} d z
\end{aligned}
$$

where the last equality was obtained using Parserval. By Lewis (2001) and Lipton (2002), we know that the option value $(V)$ of an European call with maturity $T$ and strike $K_{x}$ is given by:

$$
V(x, T)=\frac{e^{-r T}}{2 \pi} \int_{i v+\mathbb{R}} e^{-i z\left(\ln \left(S_{0}\right)+(r-q) T\right)} e^{T \psi_{\beta}(-z)} \frac{-K_{x}^{i z+1}}{z(z-i)} d z,
$$

with $v \in(a, b)$ and real constants $a<-1$ and $b>0$, where $K_{x}=S_{0} e^{(r-q) T} e^{x}$. Rewriting, we have

$$
V(x, T)=\frac{-K_{x} e^{-r T}}{2 \pi} \int_{i v+\mathbb{R}} e^{i z x} \frac{e^{T \psi_{\beta}(-z)}}{z(z-i)} d z, \quad v>1 .
$$

Observe that

$$
\begin{aligned}
\frac{\partial B S_{c}\left(K, \sigma_{i m p}(x, \beta)\right)}{\partial x} & =\frac{\partial B S_{c}\left(K, \sigma_{i m p}(x, \beta)\right)}{\partial K} \frac{\partial K}{\partial x}+\frac{\partial B S_{c}\left(K, \sigma_{i m p}(x, \beta)\right)}{\partial \sigma} \frac{\partial \sigma}{\partial x} \\
& =-N\left(d_{2}\right) K e^{-r T}+\frac{\partial B S_{c}\left(K, \sigma_{i m p}(x, \beta)\right)}{\partial \sigma} \frac{\partial \sigma}{\partial x} \\
& =\frac{\partial V}{\partial x}(x, T) .
\end{aligned}
$$

Where the last equality is obtained using (15). Then,

$$
\operatorname{sgn}\left(\frac{\partial \sigma_{i m p}}{\partial x}(x, \beta)\right)=\operatorname{sgn}\left(\frac{\partial V}{\partial x}(x, T)+N\left(d_{2}(x)\right) K e^{-r T}\right),
$$

now deriving (15) w.r.t $x$, we have:

$$
\frac{\partial V}{\partial x}(x, T)=\frac{K e^{-r T}}{2 \pi} \int_{i v+\mathbb{R}} e^{i z x} \frac{1}{i z} e^{T \psi_{\beta}(-z)} d z, \quad v>1 .
$$

Then,

$$
\operatorname{sgn}\left(\frac{\partial \sigma_{i m p}}{\partial x}(x, \beta)\right)=\operatorname{sgn}\left(\frac{1}{2 \pi} \int_{i v+\mathbb{R}} e^{i z x} \frac{1}{i z} e^{T \psi_{\beta}(-z)} d z+N\left(d_{2}(x)\right)\right), \forall x,
$$


when $x=0$,

$$
\operatorname{sgn}\left(\frac{\partial \sigma_{i m p}}{\partial x}(0, \beta)\right)=\operatorname{sgn}\left(\frac{1}{2 \pi} \int_{i v+\mathbb{R}} \frac{1}{i z} e^{T \psi_{\beta}(-z)} d z+N\left(-\frac{\sigma \sqrt{T}}{2}\right)\right) .
$$

Now we know by a result due to Fajardo and Mordecki (2006) that in symmetric Lévy markets $(\beta=-0.5)$, the implied volatility must be symmetric w.r.t to the log-moneyness i.e. $\frac{\partial \sigma_{i m p}}{\partial x}(0,-0.5)=0$, Then

$$
\frac{1}{2 \pi} \int_{i v+\mathbb{R}} \frac{1}{i z} e^{T \psi_{-0.5}(-z)} d z=-N\left(-\frac{\sigma \sqrt{T}}{2}\right) .
$$

Finally, remember that the price $f_{x}$ of a digital call option with barrier $K_{x}$, computed in our Lévy market can be expressed as ${ }^{2}$,

$$
f_{x}=-\frac{e^{-r T}}{2 \pi} \int_{i v+\mathbb{R}} e^{i z x} \frac{1}{i z} e^{T \psi_{\beta}(-z)} d z, \forall x, \quad v>0,
$$

From here the result follows and the put case is an immediate consequence of $g_{x}=e^{-r T}-f_{x}$.

Now under symmetry we can price other barrier options as asset-or- nothing barrier options, i.e. at maturity derivative pays off $p\left(S_{T}\right)=S_{T} 1_{\left\{S_{T}>K\right\}}$.

Corollary 3.1. Under symmetry the price $p$ of an asset-or-nothing option with barrier $S_{0}^{2}$ is given by:

$$
p=e^{-r T} S_{0}\left[1-N\left(-\frac{\sigma \sqrt{T}}{2}\right)\right] .
$$

Proof. Using Corollary 2.14 in Carr and Lee (2009) with the martingale $S_{t}=\exp \left(X_{t}+\ln \left(S_{0}\right)+(r-\delta) t\right)$ and $H=1$, we have:

$$
E\left[S_{0} 1_{\left\{S_{T}<1\right\}}\right]=E\left[S_{T} 1_{\left\{S_{T} \geq S_{0}^{2}\right\}}\right]
$$

and by Th. 3.1, we have

$$
p=e^{-r T} E\left[S_{T} 1_{\left\{S_{T} \geq S_{0}^{2}\right\}}\right]=S_{0} e^{-r T}\left[1-N\left(-\frac{\sigma \sqrt{T}}{2}\right)\right]
$$

\footnotetext{
${ }^{2}$ See Eberlein, Glau, and Papapantoleon (2010).
} 


\section{Absence of Symmetry}

\subsection{Approximations}

Now denote by $I(\beta, x)$ the integral defined in (16). Let $I_{\beta}$ and $I_{x}$ denote the partial derivatives of $I(\beta, x)$ with respect to $\beta$ and $x$. Then some approximations can be obtained.

Corollary 4.1. If we consider not too asymmetric markets $(|\beta+0.5|<\epsilon)$ and near at-the-money digital call options $(|x|<\varepsilon)$.

- For a barrier $K_{x}$, we have

$$
I(-0.5, x) \approx e^{-r T} N\left(-\frac{\sigma \sqrt{T}}{2}\right)+x I_{x}(-0.5,0)
$$

- For any symmetry parameter $\beta$, we have:

$$
I(\beta, 0) \approx e^{-r T} N\left(-\frac{\sigma \sqrt{T}}{2}\right)+(\beta+0.5) I_{\beta}(-0.5,0)
$$

- In general, we have:

$$
I(\beta, x) \approx e^{-r T} N\left(-\frac{\sigma \sqrt{T}}{2}\right)+(\beta+0.5) I_{\beta}(-0.5,0)+x I_{x}(-0.5,0)
$$

Proof. It follows directly from Taylor approximations and our Theorem 3.1.

Similar approximations can be obtained for the digital put option, since $g_{x}=e^{-r T}-I(\beta, x)$.

Observe that in the approximations we need to compute:

$$
I_{\beta}(-0.5,0)=\frac{T e^{-r T}}{2 \pi} \int_{i v+\mathbb{R}} \frac{1}{i z} e^{T \psi_{-0.5}(-z)} \frac{\partial \psi_{-0.5}}{\partial \beta}(-z) d z, v>0
$$

and

$$
I_{x}(-0.5,0)=\frac{e^{-r T}}{2 \pi} \int_{i v+\mathbb{R}} e^{T \psi_{-0.5}(-z)} d z, v>0
$$

then we need a specific expression for the characteristic exponent and using FFT techniques we can compute the integrals, as we will present in Section 5 . 


\subsection{More General Results}

First, we need to extend Th. 7.6 in Carr and Lee (2009).

Theorem 4.1. Let $X_{t}$ be a Lévy process with characteristic triplet $(\mu, \sigma, \Pi)$ and characteristic exponent denoted by $\psi$, with Lévy measure given by $\Pi(d y)=$ $e^{\beta y} \Pi_{0}(d y)$, where $\beta \neq-0.5$ (absence of symmetry) and define

$$
\alpha:= \begin{cases}-\frac{\psi(2 \beta i)}{2 \beta}, & \beta \neq 0 \\ \mu, & \beta=0 .\end{cases}
$$

Then for any payoff function $!^{3} f$, we have:

$$
E f\left(S_{T}\right)=E\left[\left(\frac{S_{T}}{S_{0} e^{\alpha T}}\right)^{-2 \beta} f\left(\frac{S_{0}^{2} e^{2 \alpha T}}{S_{T}}\right)\right]
$$

Proof. Here we follow Carr and Lee (2009), i.e, define the following process:

$$
Y_{t}:=-2 \beta X_{t}+(T-t) \psi(2 \beta i) .
$$

Denote by $\Pi_{y}$ its Lévy measure. Then,

$$
\Pi_{y}(x)=e^{-\left(\beta+\frac{1}{2}\right)} \Pi_{x}(d x)=e^{-\frac{1}{2}} \prod_{0}(d x),
$$

as Fajardo and Mordecki (2006) proved, process $Y_{t}$ satisfy market symmetry property. Moreover, as $E\left(e^{-2 \beta X_{t}}\right)=e^{t \psi(2 \beta i)}<\infty$, the price process $\widetilde{S}_{t}=S_{0}^{-2 \beta} e^{Y_{t}}=\left(S_{t} e^{\alpha(T-t)}\right)^{-2 \beta}$ is a martingale and satisfy the desired symmetry property.

Finally, using our symmetric process $\widetilde{S}_{t}$, we can apply Th. 2.5 in Carr and Lee (2009) to the function $x \mapsto f\left(x^{-1 / 2 \beta}\right)$, from here the result follows.

\section{$5 \quad$ Numerical Examples}

Lets consider the Normal Inverse Gaussian(NIG) distribution. Which has the following characteristic function:

$\psi_{\beta}(z)=i z \mu+\delta\left(\sqrt{\alpha^{2}-\beta^{2}}-\sqrt{\alpha^{2}-(\beta+i z)^{2}}\right),-\beta-\alpha<\operatorname{Im}(z)<\beta+\alpha$.

\footnotetext{
${ }^{3} \mathrm{~A}$ payoff function is a nonnegative Borel function on $\mathbb{R}$.
} 
From here

$$
\frac{\partial \psi_{\beta}}{\partial \beta}(z)=\delta\left(\frac{\beta+i z}{\sqrt{\alpha^{2}-(\beta+i z)^{2}}}-\frac{\beta}{\sqrt{\alpha^{2}-\beta^{2}}}\right)
$$

Before pricing Barrier options we need to estimate the parameters of the NIG distribution. To this end we consider daily returns of S\&P500 from $12 / 01 / 2009$ to $12 / 01 / 2011$ and using maximum likelihood estimation we find $(\mu, \alpha, \delta, \beta)=(0.0018,49.99,0.0085,-9.22)$. But we need the risk-neutral parameters, then we use the density given by the Esscher Transform. To compute this density we need the interest rate so we use the interest rate given by the U.S. Treasury in that date $r=0.0012$. Under this transformation we obtain the following parameters:

$$
\left(\mu^{*}, \alpha^{*}, \delta^{*}, \beta^{*}\right)=(0.0018,49.99,0.0085,-4.18)
$$

With these parameters and the expressions (20) and (21), we can compute the integrals given in (17) and (18) in the overlap of the strips giving $I_{\beta}(-0.5,0)=0.2621$ and $I_{x}(-0.5,0)=7.3212$.

Now we want to price barrier options on S\&P500 that matures in one year, i.e. $T=1$. We need the volatility then we use the VIX of $12 / 01 / 11$, as a proxy of S\&P500 volatility, $\sigma=0.2741$.

\subsection{Call and put digital option}

Using our Th. 3.1 we know that he price of a call and put digital option ATM under symmetry is given by:

$$
f_{0}=e^{-0.0012} N(-0.1371)=0.4449,
$$

and

$$
g_{0}=e^{-0.0012}[1-N(-0.1371)]=0.5539
$$

and the approximations are given by:

- For any barrier $K_{x}$, such $x \in[-\varepsilon, \varepsilon]$ we have

$$
I(-0.5, x) \approx 0.4449+7.3212 x
$$

- For any symmetry parameter $\beta \in[-0.5-\epsilon,-0.5+\epsilon]$, we have:

$$
I(\beta, 0) \approx 0.4449+0.2621(\beta+0.5)
$$


- In general, we have:

$$
I(\beta, x) \approx 0.4449+0.2621(\beta+0.5)+7.3212 x
$$

Now using $\varepsilon=\epsilon=0.01$, we have the following figure.

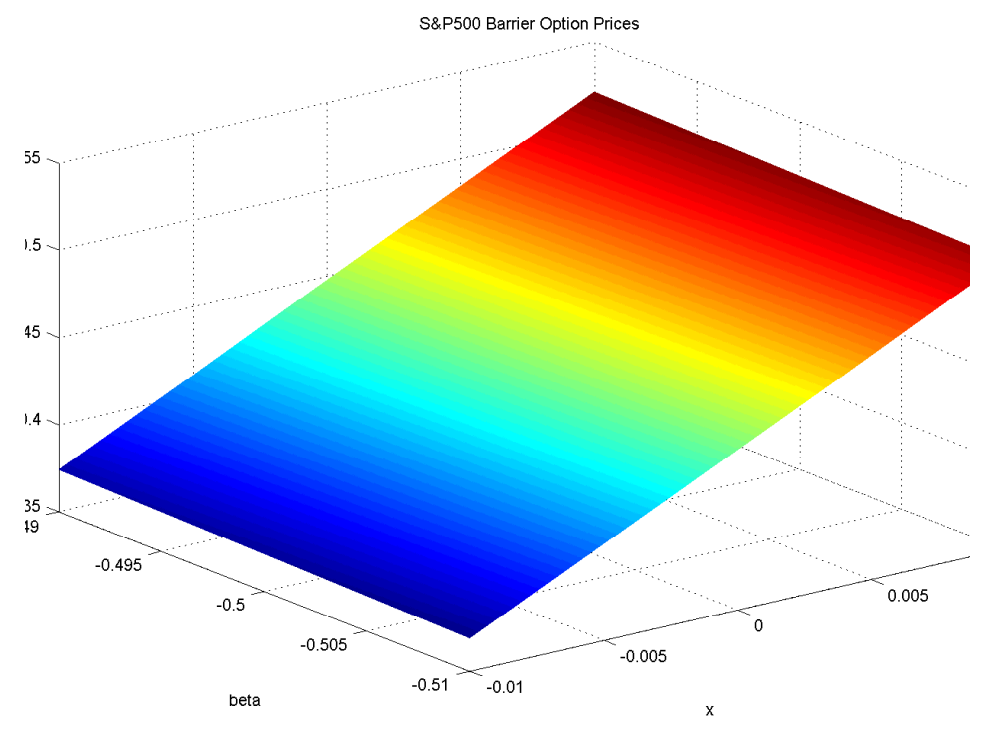

Figure 1: Barrier Option Prices for different Moneynesss and Symmetries 


\subsection{Down-and-in power option}

Now to price a down-and-in power option on S\&P500 we observe that in the

NIG case we have:

$$
\alpha=\mu=0.0018 \text {. }
$$

Using $T=1$ and applying Th. 4.1 to the function $f\left(S_{T}\right)=1_{\left\{S_{T}>1\right\}}$, we obtain:

$$
\begin{gathered}
E\left(1_{\left\{S_{T}>1\right\}}\right)=E\left(1_{\left\{S_{T}^{8.36}>1\right\}}\right)=E\left[\left(\frac{S_{T}}{S_{0} e^{0.0018}}\right)^{8.36} 1_{\left\{\frac{S_{0}^{2} e^{0.0036}}{S_{T}}>1\right\}}\right] \\
E\left(1_{\left\{\widetilde{S}_{T}>1\right\}}\right)=E\left[\left(\frac{\left(S_{T}^{*}\right)^{8.36}}{e^{0.015}}\right) 1_{\left.\left\{\frac{1444.23 e^{0.0036}}{S_{T}^{*}}>1\right\}\right]}\right]
\end{gathered}
$$

Where $\widetilde{S}_{T}=S_{T}^{8.36}$ satisfies symmetry properties and $S_{T}^{*}=e^{X_{T}}$ is an asymmetric process. Using Th. 3.1, we obtain:

$$
e^{-0.0012} E\left[\left(S_{T}^{*}\right)^{8.36} 1_{\left\{1449.4>S_{T}^{*}\right\}}\right]=0.4449 * e^{0.015}=0.4516
$$

\section{Conclusions}

We have obtained an easy way to compute the price some barrier options under Lévy processes, using the symmetry property obtained by Fajardo and Mordecki (2006) and in case of absence of symmetry we have obtained some approximations and extending Th. 7.6 in Carr and Lee (2009) we have obtained a general relationship.

Some possible extensions are of interest, as the pricing of the class of Contingent Convertible with Cancelable Coupons (CoCa CoCos) introduced by Corcuera, Fajardo, Jonsson, Schoutens, and Valdivia (2013) under Lévy processes.

\section{References}

Asmussen, S., F. Avram, and M. Pistorius (2004): "Russian and American put options under exponential phase-type Lévy models," Stochast. Process. Applic., 109, 79-111. 
Asmussen, S., D. Madan, and M. Pistorius (2007): "Pricing equity default swap under an approximation of the CGMY Lévy model," J. Compt. Finance, 11(2), 79-93.

Bates, D. (1997): "The skewness premium: Option Pricing under Asymmetric Processes," Advances in Futures and Options Research, 9, 51-82.

Carr, P., And J. Crosby (2010): "A Class of Levy Process Models with almost exact calibration of both barrier and vanilla FX options," Quantitative Finance, 10(10), 1115-1136.

Carr, P., K. Ellis, and V. Gupta (1998): "Static Hedging of Exotic Options," Journal of Finance, 53(3), 1165-1190.

Carr, P., And R. Lee (2009): "Put Call Symmetry: Extensions and Applications," Math. Finance., 19(4), 523-560.

Carr, P., And L. Wu (2007): "Stochastic Skew in Currency Options," Journal of Financial Economics, 86(1), 213-247.

Cont, R., And P. Tankov (2004): Financial Modelling with Jump Processes. Chapman \& Hall /CRC Financial Mathematics Series.

Corcuera, J., J. Fajardo, H. Jonsson, W. Schoutens, and A. ValDiviA (2013): "Close Form Pricing Formulas for CoCa CoCos," Available at SSRN: http://ssrn.com/abstract $=2206493$.

Eberlein, E., K. Glau, and A. Papapantoleon (2010): "Analysis of Fourier transform valuation formulas and applications," Applied Mathematical Finance, 17(3), 211-240.

- (2011): "Analyticity of the Wiener-Hopf factors and valuation of exotic options in Lévy models.," in Advanced Mathematical Methods for Finance, ed. by G. D. Nunno, and B. Øksendal. Springer Verlag.

Fajardo, J., And E. Mordecki (2006): "Symmetry and Duality in Lévy Markets," Quantitative Finance, 6(3), 219-227.

(2008): "Duality and Derivative Pricing with Time-Changed Lévy processes," Brazilian Review of Econometrics, 28(1), 95-110. 
(2010): "Market Symmetry in Time Changed Brownian Models," Finance Research Letters, 7(1), 53-59.

(2012): "Skewness Premium with Lévy Processes," Forthcoming Quantitative Finance.

Jacod, J., And A. ShiRyaev (1987): Limit Theorems for Stochastic Processes. Springer, Berlin, Heidelberg.

Kou, S., and H. Wang (2004): "Option pricing Under a Double Exponential Jump Diffusion Model," Management Science, 50(9), 1178-1192.

Lewis, A. L. (2001): "A simple option formula for general jump-diffusion and other exponential Lévy processes," Working paper. Envision Financial Systems and OptionCity.net Newport Beach, California, USA. Available at http://www.optioncity.net.

Lipton, A. (2002): "Assets with Jumps," Risk, 15, 149-153.

Molchanov, I., And M. Schmutz (2010): "Multivariate Extension of Put-Call Symmetry.," SIAM Journal on Financial Mathematics, 1, 396426.

Rogers, L. C. G. (2000): "Evaluating first-passage probabilities for spectrally one-sided Lévy processes.," J. Appl. Probab., 37, 1173-1180.

Schoutens, W. (2003): Lévy Processes in Finance: Pricing Financial Derivatives. Wiley, New York.

Schoutens, W., and J. Cariboni (2009): Lévy Processes in Credit Risk. Wiley, New York.

Tehranchi, M. (2009): "Symmetric martingales and symmetric smiles," Stochastic Processes and their Applications, 119, 3785-3797. 